\title{
MULTI-RESOLUTION PARALLEL INTEGRAL PROJECTION FOR FAST LOCALIZATION OF A STRAIGHT ELECTRODE IN 3D ULTRASOUND IMAGES
}

\author{
Marián Uherčík ${ }^{a, b, *}$, Jan Kybic $^{a}$, Hervé Liebgott ${ }^{b}$, Christian Cachard $^{b}$ \\ ${ }^{a}$ Center for Machine Perception, Czech Technical University in Prague, Czech Republic, \\ ${ }^{b}$ CREATIS, Université de Lyon, INSA-Lyon, Université Lyon 1, CNRS UMR5220, INSERM U630, \\ F-69621, France, * E-mail: uhercik@cmp.felk.cvut.cz
}

\begin{abstract}
We address the problem of fast and accurate localization of miniature surgical instruments like needles or electrodes using 3D ultrasound (US). An algorithm based on maximizing a Parallel Integral Transform (PIP) can automatically localize line-shaped objects in 3D US images with accuracy on the order of hundreds of micrometers. Here we propose to use a multi-resolution to accelerate the algorithm significantly. We use a maximum function for downsampling to preserve the high intensity voxels of a thin electrode. We integrate the multi-resolution pyramid into a hierarchical mesh-grid search of PIP. The experiments with a tissue mimicking phantom and breast biopsy data show that proposed method works well on real US images. The speed-up is threefold compared to original PIP method with the same accuracy $0.4 \mathrm{~mm}$. A further speed-up up to 16 times is reached by an early stopping of the optimization, at the expense of some loss of accuracy.
\end{abstract}

Index Terms - 3D ultrasound, electrode, localization, parallel integral projection, multi-resolution

\section{INTRODUCTION}

Tool localization using medical imaging modalities such as MRI, CT and US allows the physician to view anatomical information of human body together with the location of surgical instruments during the intervention [1]. We focus on the 3D US modality which is relatively affordable, non-invasive, and involves no ionizing radiation. It offers real-time 3D images with sufficient resolution, but with large amount of speckle noise and acoustic artifacts.

Our task is to automatically localize an electrode or a thin needle inserted into a biological tissue. Its diameter is about 1 millimeter or smaller. The electrode is usually straight and appears as high intensity voxels in a US image. One possible application is finding a plane passing through the electrode for visualization purposes, as physicians are used to examine 2D US images. Other applications include automatic tool guidance or tracking neuronal recording sites.

\subsection{Previous work}

A variety of algorithms for object localization in US data have been proposed. The position of an electrode axis can be determined in a 2D image using Principal Component Analysis [2] on a thresholded variance image. Ding [3] proposes to find the lines in 2D projections of a volume by a parallel projection. Barva $[4,5]$ shows that 3D Parallel Integral Projection can be used to find the electrode axis. Novotny [6] decomposes the volume to overlapping spheres and finds the instrument in each subvolume. He uses a modified Radon Transform implemented on a GPU. Barva $[4,7]$ uses a polynomial to describe a deformed electrode, whose parameters are estimated by a RANSAC algorithm.

\section{PARALleL INTEGRAL PROJECTION (PIP)}

Our method is based on a Parallel Integral Projection (PIP) for electrode localization $[4,5]$ which works in two steps: (i) axis localization, and (ii) tip localization. We assume that the axis is straight and the length is much greater than the diameter.

The PIP $[4,5]$ is a transform that maps an image function $\mathcal{I}: \mathbb{R}^{3} \rightarrow \mathbb{R}$ representing volume data to a function $\mathcal{P}_{\mathcal{I}}$ : $\mathbb{R}^{4} \rightarrow \mathbb{R}$. Formally, the PIP transformation of $\mathcal{I}(\mathbf{x})$ is:

$$
\mathcal{P}_{\mathcal{I}}(u, v, \alpha, \beta)=\int_{-\infty}^{\infty} \mathcal{I}\left(\mathbf{R}(\alpha, \beta) \cdot(u, v, \tau)^{T}\right) \mathrm{d} \tau,
$$

where $\mathbf{R}(\alpha, \beta)$ is a rotation matrix representing a rotation around the $x$-axis by angle $\alpha$, and around $y$-axis by angle $\beta$. The PIP transform is similar to the Radon transform.

To find the electrode axis we need to find the maximum of $\mathcal{P}_{\mathcal{I}}$. We decompose the maximization of $P_{I}(u, v, \alpha, \beta)$ to an inner maximization with respect to $(u, v)$ and an outer maximization with respect to $(\alpha, \beta)$, using a hierarchical meshgrid approach [8].

We find an electrode endpoint along the estimated axis $[4,5]$ as point where intensity first decreases under a threshold $T_{1}$, estimated using probability density of intensities of the electrode $P(e l)$ and background $P(b g)$. We skip breaks shorter than a threshold $T_{2}$, which is estimated as a $95 \%$ quantile of the distribution of break lengths. 


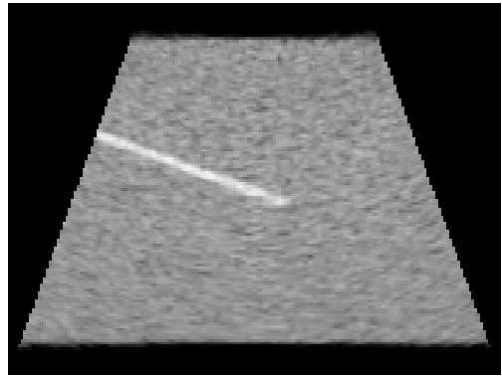

Original image

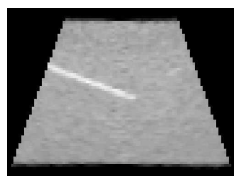

$\max 2 \mathrm{x}$

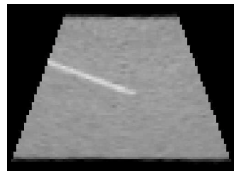

$\operatorname{avg} 2 \mathrm{x}$

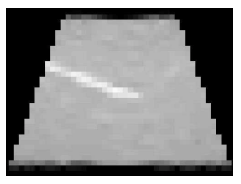

$\max 4 \mathrm{x}$

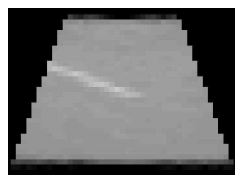

$\operatorname{avg} 4 \mathrm{x}$

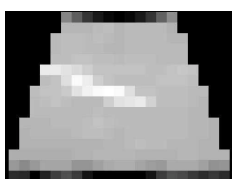

$\max 8 \mathrm{x}$

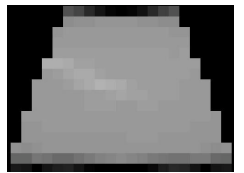

$\operatorname{avg} 8 \mathrm{x}$

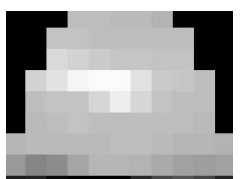

$\max 16 x$

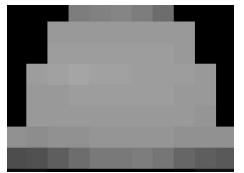

avg 16x

Fig. 1. The 2D image of slice with needle selected from 3D volume, and multiple downsampled images using max and avg functions. The original resolution was $53 \times 71 \times 262$ voxels, for each coarser level the resolution has been divided by two. You can see the electrode of radius $0.3 \mathrm{~mm}$ is blurred out in case of the avg function in coarse resolutions, while for the max function the contrast between electrode and background stays relatively good.

\section{MULTI-RESOLUTION PARALLEL INTEGRAL PROJECTION (MR PIP)}

As the original PIP method $[4,5]$ is rather slow, we want to improve its speed. We propose to use downsampled 3D images for electrode localization. We will show that using a maximum function we obtain reliable results on downsampled images better than with standard downsampling based on averaging.

A discrete function $\mathcal{I}_{s m p}: \mathbb{N}^{3} \rightarrow \mathbb{R}$ represents an image $\mathcal{I}$ at the pixel grid. We define the downsampled image $\mathcal{I}_{\text {down }}$ of image $\mathcal{I}_{s m p}$ by a factor $M_{x}, M_{y}, N_{z} \in \mathbb{N}$ :

$$
\mathcal{I}_{\text {down }}^{f}(x, y, z)=f\left\{\mathcal{I}_{\text {smp }}\left(x M_{x}+i, y M_{y}+j, z M_{z}+k\right)\right\},
$$

where $0 \leq i<M_{x}, 0 \leq j<M_{y}, 0 \leq k<M_{z}$. We use a function $f: \mathbb{R}^{n} \rightarrow \mathbb{R}$ to filter the set of neighboring voxels, yielding a pixel value at the coarser resolution. $f$ should preserve well the differences between the electrode and the background. As $f$, we will use $a v g$ for average and $\max$ for maximum of a set of values. We set $M_{x}=M_{y}=M_{z}=$ 2. A multi-resolution pyramid was constructed by repeated downsampling (Figure 1):

$$
\mathcal{I}_{1}=\mathcal{I}_{\text {smp }}, \mathcal{I}_{2}=\mathcal{I}_{\text {down }}^{\text {max }}\left(\mathcal{I}_{1}\right), \ldots \mathcal{I}_{n}=\mathcal{I}_{\text {down }}^{\max }\left(\mathcal{I}_{n-1}\right) .
$$

\subsection{Algorithm}

We use a hierarchical mesh-grid search $[4,5,8]$ for finding the maximum $\left(\alpha_{\max }, \beta_{\max }, u_{\max }, v_{\max }\right)$ of the PIP transform with respect to $\arg \max _{\alpha, \beta} \max _{u, v} \mathcal{P}_{\mathcal{I}}(u, v, \alpha, \beta)$. We modify this maximization using multi-resolution (Algorithm 1). The discretization step $\Delta$ for $(\alpha, \beta)$ and the discretization step $\Gamma$ for $(u, v)$ is iteratively decreased as the resolution level $\mathcal{I}_{k}$ is refined. This makes the method faster than PIP.

By stopping the iterative algorithm early we further accelerate the algorithm. We stop on a coarse resolution $\mathcal{I}_{K_{\text {final }}}$ and set larger discretization steps $\Delta_{\text {final }}$ and $\Gamma_{\text {final }}$. This usually amounts to using a smaller number of iterations but

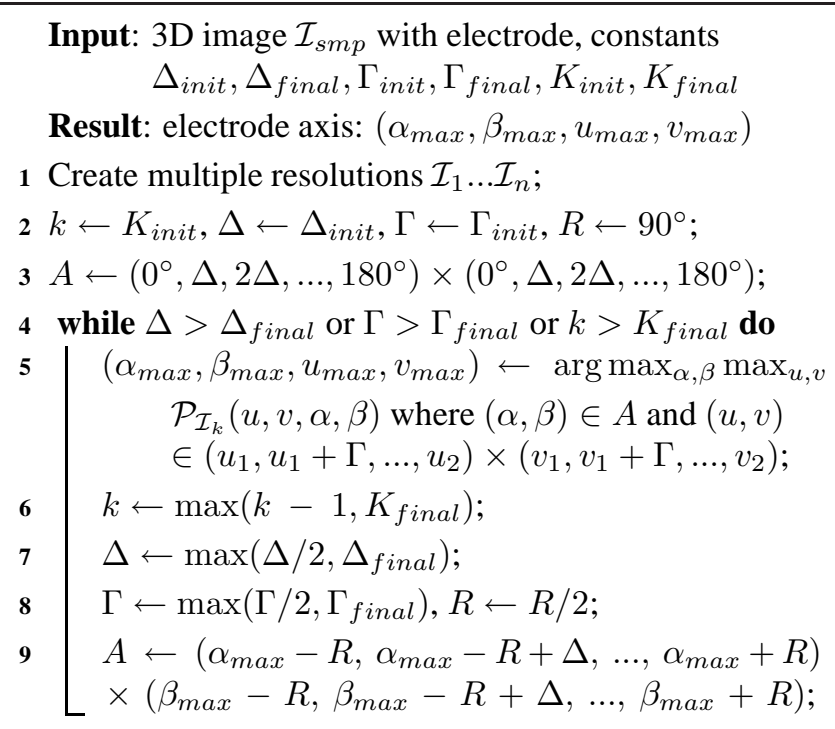

Algorithm 1: Hierarchical mesh-grid search of MR PIP. Constants $u_{1}, u_{2}, v_{1}, v_{2}$ are boundaries of the projected volume, $A$ contains the set of angles for evaluation of $\mathcal{P}_{\mathcal{I}_{k}}, R$ is the size of the current interval of angles. $\Delta_{\text {init }}, \Delta_{\text {final }}$ and $\Gamma_{\text {init }}, \Gamma_{\text {final }}$ are initial and final discretization steps for $\Delta$ and $\Gamma$ respectively. $K_{\text {init }}$ and $K_{\text {final }}$ is initial and final level of resolution.

decreases the accuracy. We call this method Fast Multiresolution Parallel Integral Projection (Fast MR PIP).

Let us analyze the time complexity of MR PIP. Number of iterations of while loop in Algorithm 1 is at most $N=\max \left\{N_{1}, N_{2}, N_{3}\right\}$ where $N_{1}=\left\lceil\log _{2} \frac{2 \Delta_{\text {init }}}{\Delta_{\text {final }}}\right\rceil, N_{2}=$ $\left\lceil\log _{2} \frac{2 \Gamma_{\text {init }}}{\Gamma_{\text {final }}}\right\rceil$ and $N_{3}=K_{\text {init }}-K_{\text {final }}+1$. Number of evaluations of $\mathcal{P}_{\mathcal{I}_{k}}$ in each iteration is dependent on maximization term in line 5. There are $T_{1}=\left(\frac{180^{\circ}}{\Delta_{\text {init }}}\right)^{2}$ evaluations of outer part maximizing over $(\alpha, \beta)$ and $T_{2}=\sum_{i=\Gamma_{\text {init }}}^{\Gamma_{\text {final }}}\left(\frac{\text { size }}{\Gamma_{i}}\right)^{2}=$ $\sqrt[4]{3} \cdot\left(\frac{\text { size }}{\Gamma_{\text {final }}}\right)^{2}$ evaluations of inner part maximizing over $(u, v)$. The total number of evaluations of $\mathcal{P}_{\mathcal{I}}(u, v, \alpha, \beta)$ 


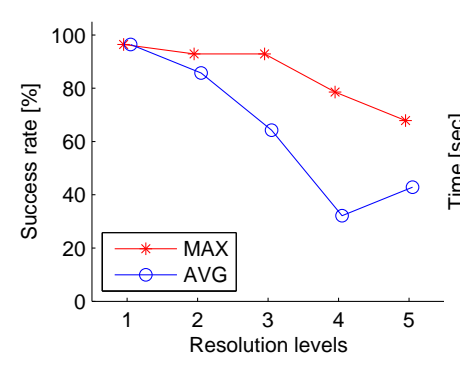

(a)

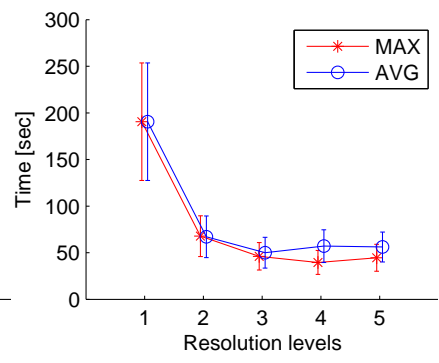

(b)

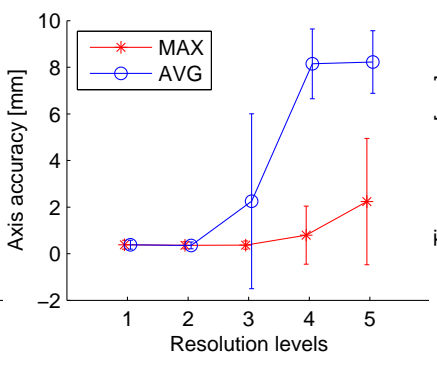

(c)

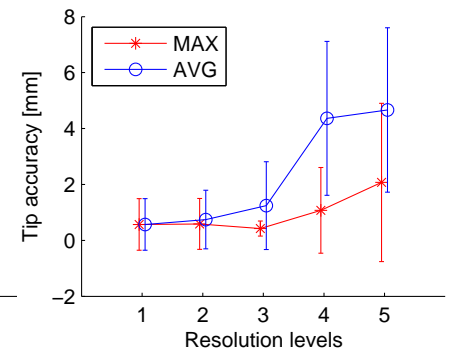

(d)

Fig. 2. Results from MR PIP on simulated data with an electrode. The original resolution of the data was $53 \times 71 \times 307$ voxels. Original PIP algorithm is equivalent to resolution level 1 . The success rate considers good results with axis accuracy better than $10 \mathrm{~mm}$. The charts show means as points and standard deviations as vertical bars.

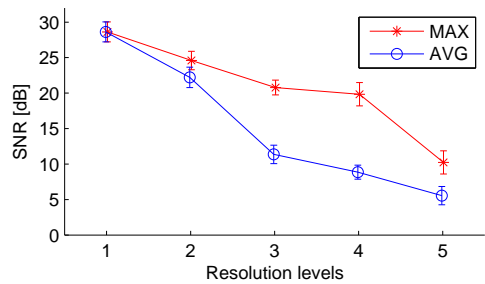

Fig. 3. Comparison of SNR of simulated data on different resolution levels for $\max$ and avg filtering function.

is $N \cdot T_{1} \cdot T_{2}$ and it depends on the size of volume and discretization steps $\Delta_{\text {init }}, \Delta_{\text {final }}, \Gamma_{\text {init }}, \Gamma_{\text {final }}$.

\section{RESULTS}

We will show that the MR PIP method is as accurate as the original PIP method while being faster. The method was implemented in MATLAB. We did all tests on a Gentoo Linux computer with a 64-bit Intel Core 2 processor at $2400 \mathrm{MHz}$.

Two measures are used to quantify the accuracy $[4,5]$. The first measure $\varepsilon_{t i p}=\|T-\hat{T}\|$ evaluates the tip localization accuracy, where $T$ is the true electrode tip, $\hat{T}$ an estimated tip and $\|\cdot\|$ is the Euclidean distance. Axis localization accuracy is given by $\varepsilon_{\text {axis }}=\max \left\{\left\|E-Q_{1}\right\|,\left\|T-Q_{2}\right\|\right\}$, where $E$ is the intercept point, $Q_{1}$ and $Q_{2}$ are the orthogonal projections of $E$ and $T$ on the estimated axis with respect to the true axis.

For evaluation of image quality we define the signal-tonoise ratio: $\mathrm{SNR}=10 \log \frac{E\left[x_{e l}^{2}\right]}{E\left[x_{b g}^{2}\right]}[\mathrm{dB}]$ where $x_{e l}$ are voxels with distance from the axis less than an electrode radius and the remaining voxels are considered as background $x_{b g}$.

\subsection{Evaluation of parameters influence}

The experiments were done on 28 simulated datasets of size $53 \times 71 \times 307$ voxels with varying electrode translation and rotation. Simulated data were generated using the US simulator FIELD II [9], set to imitate the US scanner Voluson 530D. The discretization parameters were fixed to $\Delta_{\text {init }}=32^{\circ}$,

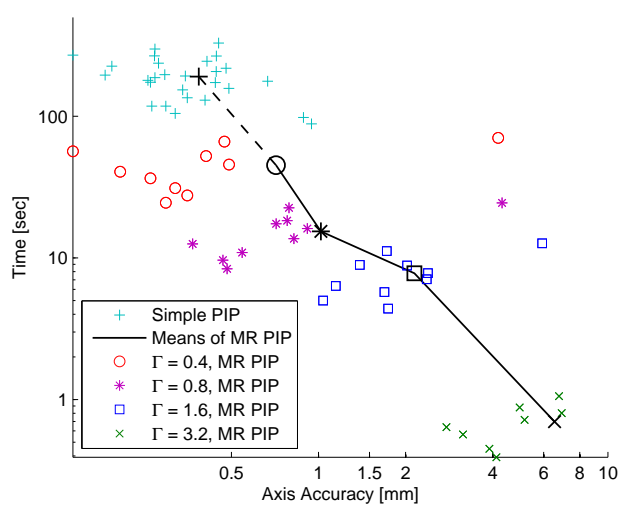

Fig. 4. The charts show the dependence of time and accuracy on final discretization $\Gamma_{\text {final }}$ for Fast MR PIP and simple PIP without any multi-resolution on simulated data.

$\Delta_{\text {final }}=1^{\circ}, \Gamma_{\text {init }}=0.4 \cdot 2^{\text {levels-1 }} \mathrm{mm}, \Gamma_{\text {final }}=0.4 \mathrm{~mm}$ where levels is number of resolutions.

We compared the max and avg function for downsampling. The SNR values of simulated data are in Figure 3. Downsampling using a $\max$ function seems to preserve the electrode shape better and also the SNR is better. Figure 2a shows the success rate for the MR PIP on synthetic data. The success rate is steadily close to $100 \%$ for the $\max$ function, and decreasing to $0 \%$ for the avg. We decided to use the $\max$ function for downsampling in the rest of experiments.

We varied the number of resolution levels from 1 to 5 and measured the time (Figure 2b). As expected, the time has been significantly reduced from the 123 seconds mean time for the original single resolution PIP method (Table 1, row 1). Best mean time was 46 seconds for resolution 3 (Table 1, row 2) with the accuracy $0.4 \mathrm{~mm}$ which is satisfactory (Figure $2 \mathrm{c}, 2 \mathrm{~d})$.

We have also evaluated the tradeoff between speed and accuracy for the Fast MR PIP method. The results for different values of the final discretization step are shown in Figure 4. We reached the mean time 7.8 seconds with axis accuracy around $2 \mathrm{~mm}$ for $\Gamma_{\text {final }}=1.6 \mathrm{~mm}$ (Table 1, row 3$)$. 


\begin{tabular}{|l|c|c|c|}
\hline data / method & time [sec] & axis ac. [mm] & tip ac. [mm] \\
\hline \hline simulated / 1 & $123 \pm 5.8$ & $0.327 \pm 0.180$ & $1.732 \pm 5.132$ \\
\hline simulated / 2 & $46.1 \pm 15$ & $0.371 \pm 0.178$ & $0.422 \pm 0.268$ \\
\hline simulated / 3 & $7.8 \pm 2.6$ & $2.143 \pm 1.369$ & $13.89 \pm 6.67$ \\
\hline \hline phantom / 2 & $62.5 \pm 26$ & $0.443 \pm 0.206$ & $0.508 \pm 0.175$ \\
\hline phantom / 3 & $7.3 \pm 2.1$ & $1.421 \pm 0.429$ & $9.335 \pm 7.747$ \\
\hline \hline br. biopsy / 2 & 61 & 0.108 & 0.569 \\
\hline br. biopsy / 3 & 5.5 & 3.270 & 3.302 \\
\hline
\end{tabular}

Table 1. Results on various data: simulated data (28 datasets), cryogel phantom ( 8 datasets), breast biopsy (1 dataset). Methods used is this table: 1 - PIP on single full resolution, 2 MR PIP with 3 resolutions, 3 - Fast MR PIP. The mean and standard deviations of elapsed time, axis accuracy and tip accuracy are reported.

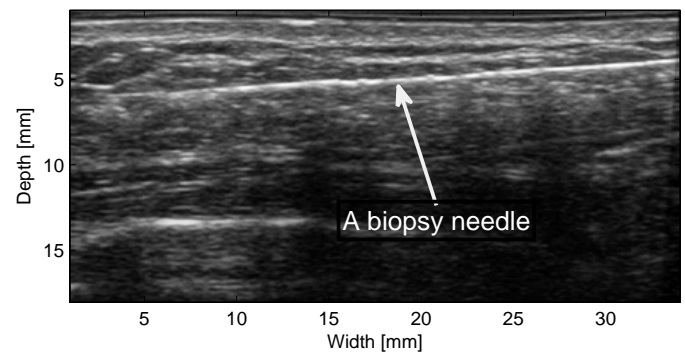

Fig. 5. Data of breast biopsy with a needle of diameter $1 \mathrm{~mm}$. The slice with needle is shown, resolution $207 \times 383 \times 208$.

\subsection{Experiments on phantom and real data}

We acquired 8 datasets of a cryogel tissue mimicking phantom with a thin tungsten electrode of $250 \mu \mathrm{m}$ in diameter and length $20 \mathrm{~mm}$ using an US scanner Voluson 530D [10]. The datasets are sector volumes of $40^{\circ} \times 40^{\circ} \times$ depth $6.2 \mathrm{~cm}$ with a resolution of $53 \times 71 \times 310$ voxels. The success rate for experiments on this data was $100 \%$ in all cases and measured time and accuracy can be found in Table 1 (row 4 for MR PIP and row 5 for Fast MR PIP).

A dataset of breast biopsy was acquired by a 3D US scanner GE Voluson E8 with the $12 \mathrm{MHz}$ probe. The biopsy needle was $1.092 \mathrm{~mm}$ in diameter. The geometry of this volume was $30^{\circ} \times 38 \mathrm{~mm}$ width $\times 19 \mathrm{~mm}$ depth with resolution $207 \times 383 \times 208$ voxels. The localization was successful with a discretization step $\Gamma_{\text {final }}=0.3 \mathrm{~mm}$ and the result matches with visual identification. The time and achieved accuracy are in Table 1 (row 6 for MR PIP and row 7 for Fast MR PIP).

\section{CONCLUSIONS}

We have presented a multi-resolution PIP method for electrode axis localization in 3D US data which is much faster than the original PIP and yet has the same accuracy. This makes PIP method final practical for real applications. Further speed-up is achieved by Fast multi-resolution PIP based on an early stopping of the hierarchical search algorithm. Rewriting the method in a compiled language or implementing the method on a GPU will also accelerate it substantially.

We propose downsampling with the $\max$ function which preserves electrode voxels better than averaging. This can be used also for other applications requiring fast detection of thin lines, e.g. vessel segmentation in a 3D image.

\section{ACKNOWLEDGEMENTS}

A dataset of breast biopsy is a courtesy of GE Medical Systems. The author Marián Uherčík was supported by an EC project MEST-CT-2005-021024 WARTHE. The author Jan Kybic was supported by Czech Ministry of Education project $1 \mathrm{M} 0567$.

\section{REFERENCES}

[1] T.M. Peters, "Image-guidance for surgical procedures," Physics in Medicine and Biology, vol. 51, no. 14, pp. R505R540, 2006.

[2] K.J. Draper, C.C Blake, D.B. Gowman, L. nad Downey, and A. Fenster, "An algorithm for automatic needle localization in ultrasound-guided breast biopsies," Medical Physics, vol. 27, pp. 1971-1979, 2000.

[3] M. Ding, H. N. Cardinal, W. Guan, and A. Fenster, "Automatic needle segmentation in 3D ultrasound images," in Proceedings of SPIE. May 2002, vol. 4681, pp. 65-76, International Society for Optical Engineering.

[4] M. Barva, Localization of Surgical Instruments in 3D Ultrasound Images, PhD Thesis CTU-CMP-2007-12, Center for Machine Perception, K13133 FEE Czech Technical University, Prague, Czech Republic, June 2007.

[5] M. Barva, M. Uherčík, J.M. Mari, J. Kybic, J.R. Duhamel, H. Liebgott, V. Hlaváč, and C. Cachard, "Parallel integral projection transform for straight electrode localization in 3D ultrasound images," To appear in IEEE Transactions of Ultrasonics, Ferroelectrics and Frequency Control.

[6] P. M. Novotny, J. A. Stoll, N. V. Vasilyev, P. J. del Nido, P. E. Dupont, and R. D. Howe, "GPU based real-time instrument tracking with three dimensional ultrasound," Medical image analysis, vol. 11, no. 5, pp. 458-64, 2007.

[7] M. Barva, J. Kybic, J.M. Mari, C. Cachard, and V. Hlaváč, "Automatic localization of curvilinear object in 3D ultrasound images," SPIE International Symposium on Medical Imaging, February 2005.

[8] R. Bulirsch and J. Stoer, Introduction to Numerical Analysis, Springer-Verlag, New York, 1991.

[9] J. A. Jensen, "Field: A program for simulating ultrasound systems," in Medical and Biological Engineering and Computing, 1996, vol. 34, pp. 351-353.

[10] J-M. Mari and C. Cachard, "Acquire real-time RF digital ultrasound data from a commercial scanner," Electronic Journal "Technical Acoustics", vol. 3, January 2007. 